The Reflection of the $X$-Rays,

Messrs. Laue, Friedrich, and Knipping's remarkable photographs taken through a crystal with X-rays have opened a new field of research. Mr. W. L. Bragg has shown that much stronger photographs are produced by a grazing reflection from mica. We have recently used the latter discovery to study the reflected beam electrically. We find that it resembles ordinary $\mathrm{X}$-rays. Just like the primary rays it ionises air and helium and produces a soft radiation when it strikes metals. The variation of its ionisation in air with pressure is also similar. We have so far obtained effects as great as $I$ or 2 per cent. of the primary beam. In some cases the measurements in helium were magnified by ionisation by collision.

The same absorption of the reflected beam was produced bv aluminium, whether the rays passed through it before or after the reflection. In one case the absorption coefficient for either position was $6 \mathrm{~cm} \cdot{ }^{-1}$, whereas for the primary beam it was $9 \mathrm{~cm}^{-1}$. This indicates that the character of the rays is unaltered by reflection, but that the amount of reflection increases with the hardness.

As Prof. Bragg has shown, the behaviour of the $\mathrm{X}$-rays in connection with ionisation strongly suggests that their energy is concentrated as if they were corpuscular. Since the rays are reflected, they must be some kind of pulse with an extended wave-front, yet after reflection they retain their corpuscular character. Thus the energy of the X-rays appears to show the contrary properties of extension over a wave-front and concentration in a point. H. Moseley.

Victoria University, Manchester, January $2 \mathrm{r}$.

Emission of Particles by Heated Metals.

Referring to Prof. Valentiner's article on "New Facts in Physics," in NATURE of January 9, you note that Messrs. Reboul and Dr. Bollement have found that copper and silver eject particles at $500^{\circ} \mathrm{C}$. in a vacuum, in air, oxygen, and carbonic acid. which forms deposits on the walls of the containing vessel. Pure silver, and several alloys having a chief content of silver, were found by me to emit particles or an emanation of some sort when heated in air to a bright red, but well below their melting points. The facts were observed during an inquiry to ascertain the cause of the sudden failure of the platinum pins of porcelain teeth, and were briefly noted in The Dental Record, September, ror.

In the presence or proximity of silver at a red heat two kinds of effects on other bodies were observed: (I) a yellow to brownish-yellow staining on the surface of various porous "investments" (plaster of paris, pumice-stone, whiting, French chalk, asbestos, and mixtures of these) against which the silver or silver alloy had been heated; (2) porcelain was rendered weak and almost friable to a varying depth from the surface in contact with silver, the deteriorating effect penetrating to a depth of 3 or $4 \mathrm{~mm}$. during half an hour's heating.

Gold. platinum, copper, zinc-singly or in combination-did not produce those effects, but when silver was present the staining was copious, and the weakening or "fritting" of porcelain in at least some degree always resulted. No analysis was made, but the inference seemed well founded that in those cases silver at a bright-red heat emitted something that gave rise to the effects noted.

Eltham, S.E

Thermal Efficiency of Gas and Electricity.

OuR attention has been directed to a short paragraph in your issue of January 16 (p. $55 \mathrm{I}$ ) regarding No. 2257 , vOL. 90] the progress of electric cooking, in which the following sentence occurs:-."The present year should see a reasonably cheap and economical electric oven put on the market to compete with the everyday gascooker, which at present, on account of its low initial cost, still holds the field against the electric oven among the general public."

Please allow me to point out that it is not merely a question of comparative cost and upkeep of apparatus, but the more important point of comparative efficiency. The heat equivalent of one unit of electricity (costing at least one penny) equals 34 10 British thermal units, while the heat equivalent of 33 cubic feet of gas (costing in London one penny) equals 16,500 British thermal units. No science can obtain from a given quantity of energy or fuel more than the maximum amount of heat it produces when completely consumed, and it is therefore apparent that electricity, so far as heat production is concerned, can never economically compare with gas. W. M. MASON,

The British Commercial Gas Association, Secretary. 47 Victoria Street, Westminster, S.W., January $2 \mathrm{I}$.

\section{Research Defence Society}

IT is said that the fifth year in the life of any society is the critical period of its fortunes. The Research Defence Society was founded on January 27 , I908. To all who are interested-and who is not?in medical research, we beg you to let us say that the society has its hands full of work, and only wants more money to do more work. Much has already been done, by lectures and by distribution of literature, to bring home to people the truth about experiments on animals in this country, and the great value of them, not only to mankind, but also to the animal world. The expenses of our society are heavy; but the good results of our work are extended far and wide. We have lately opened a bureau and exhibition at I7I Piccadilly (opposite Burlington House). We are exhibiting pictures, portraits, charts, anæsthetics and inhalers, germs in pure culture, tsetse-flies and mosquitoes, and so forth. This little exhibition, every day, and all day long, displays to "the man in the street" the facts of the case. We are the only society which is doing work of this kind; but, of course, it cannot be done without money. Our record for the last four years gives us the right to hope for a great increase of our membership, and of our funds, in the coming year.

David GILl, President.

F. M. Sandwith, Hon. Treasurer. Stephen Paget, Hon. Secretary.

2 I Ladbroke Square, W., January 24

\section{Retinal Shadows?}

$I_{F}$, in the early morning, when the eyes are first opened, one looks at the white ceiling, branching lines are to be seen resembling blood-vessels. These figures only persist for about one second. It seems necessary for the eyes to be closed, and have a long rest, to show them clearly. In the daytime quite a long rest is necessary for them to be visible at all.

So far as I can judge they always have the same form. It would appear from this that they are really the shadows of the blood-vessels; but why they should only be visible for about a second after opening the eyes, and then only after a long rest, does not seem clear.

It may be that the phenomenon is well known, but, if so, I have not chanced to hear of it.

$$
\text { R. M. Deeley. }
$$

Abbeyfield, Salisbury Avenue, Harpenden, January $2 \mathrm{r}$ 